\title{
A Practical Linear Time Algorithm for Trivial Automata Model Checking of Higher-Order Recursion Schemes
}

\author{
Naoki Kobayashi \\ Tohoku University
}

\begin{abstract}
The model checking of higher-order recursion schemes has been actively studied and is now becoming a basis of higher-order program verification. We propose a new algorithm for trivial automata model checking of higher-order recursion schemes. To our knowledge, this is the first practical model checking algorithm for recursion schemes that runs in time linear in the size of the higher-order recursion scheme, under the assumption that the size of trivial automata and the largest order and arity of functions are fixed. The previous linear time algorithm was impractical due to a huge constant factor, and the only practical previous algorithm suffers from the hyper-exponential worst-case time complexity, under the same assumption. The new algorithm is remarkably simple, consisting of just two fixed-point computations. We have implemented the algorithm and confirmed that it outperforms Kobayashi's previous algorithm in a certain case.
\end{abstract}

\section{Introduction}

The model checking of higher-order recursion schemes [9]20] (higher-order model checking, for short) has been studied extensively, and recently applied to higherorder program verification 12/10/18/17/21. A higher-order recursion scheme [9]20] is a grammar for describing a possibly infinite tree, and the higher-order model checking is concerned about whether the tree described by a given higherorder recursion scheme satisfies a given property (typically expressed by the modal $\mu$-calculus or tree automata). Higher-order model checking can be considered a generalization of finite-state and pushdown model checking. Just as software model checkers for procedural languages like BLAST [4] and SLAM [3] have been constructed based on finite-state and pushdown model checking, one may hope to construct software model checkers for higher-order functional languages based on higher-order model checking. Some evidence for such a possibility has been provided recently [12/0/18/17/21].

The main obstacle in applying higher-order model checking to program verification is its extremely high worst-case complexity: $n$-EXPTIME completeness [20] (where $n$ is the order of a given higher-order recursion scheme). Kobayashi and Ong [1216] showed that, under the assumption that the size of properties and the largest order and arity of functions are fixed, the model 
checking is actually linear time in the size of the higher-order recursion scheme for the class of properties described by trivial automata [2], and polynomial time for the full modal $\mu$-calculus. Their algorithms were however of only theoretical interest; because of a huge constant factor (which is $n$-fold exponential in the other parameters), they are runnable only for recursion schemes of order 2 at highest.

The only practical algorithm known to date is Kobayashi's hybrid algorithm [10], used in the first higher-order model checker TRECS [11]. According to experiments, the algorithm runs remarkably fast in practice, considering the worst-case complexity of the problem. The worst-case complexity of the hybrid algorithm is, however, actually worse than Kobayashi's naive algorithm [12: Under the same assumption that the other parameters are fixed, the worst-case time complexity of the hybrid algorithm [10] is still hyper-exponential in the size of the recursion scheme. In fact, one can easily construct a higher-order recursion scheme for which the hybrid algorithm suffers from an $n$-EXPTIME bottleneck in the size of the recursion scheme. Thus, it remained as a question whether there is a practical algorithm that runs in time polynomial in the size of the higher-order recursion scheme. The question is highly relevant for applications to program verification [1218, as the size of a higher-order recursion scheme corresponds to the size of a program.

The present paper proposes the first (arguably) practical, linear tim 11 algorithm for trivial automata model checking of recursion schemes (i.e. the problem of deciding whether the tree generated by a given recursion scheme $\mathcal{G}$ is accepted by a given trivial automaton $\mathcal{B}$ ). Like Kobayashi and Ong's previous algorithms [1210 16], the new algorithm is based on a reduction of model checking to intersection type inference, but the algorithm has also been inspired by game semantics 201] (though the game semantics is not explicitly used). The resulting algorithm is remarkably simple, consisting of just two fixedpoint computations. We have implemented the new algorithm, and confirmed that it outperforms Kobayashi's hybrid algorithm [10] in a certain case. Another advantage of the new algorithm is that it works for non-deterministic trivial automata, unlike the hybrid algorithm (which works only for deterministic trivial automata).

Unfortunately, the current implementation of the new algorithm is significantly slower than the hybrid algorithm [10] (which already incorporates a number of optimizations) in most cases. However, the new algorithm provides a hope that, with further optimizations, one may eventually obtain a model checking algorithm that scales to large programs (because of the fixed-parameter linear time complexity).

The rest of this paper is structured as follows. Section 2 reviews higher-order recursion schemes and previous type-based model checking algorithms for recursion schemes. Section 3 presents a new model checking algorithm, and Section 4 proves the correctness of the algorithm. Section 5 reports preliminary experiments. Section [6] discusses related work and Section 7 concludes.

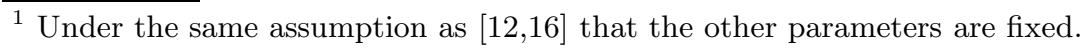




\section{Preliminaries}

We write $\operatorname{dom}(f)$ for the domain of a map $f$. We write $\widetilde{x}$ for a sequence $x_{1}, \ldots, x_{k}$, and write $\left[t_{1} / x_{1}, \ldots, t_{k} / x_{k}\right] u$ for the term obtained from $u$ by replacing $x_{1}, \ldots, x_{k}$ in $u$ with $t_{1}, \ldots, t_{k}$. A $\Sigma$-labeled tree (where $\Sigma$ is a set of symbols), written $T$, is a map from $[m]^{*}$ (where $m$ is a positive integer and $[m]=\{1, \ldots, m\}$ ) to $\Sigma$ such that (i) $\epsilon \in \operatorname{dom}(T)$, (ii) $x i \in \operatorname{dom}(T)$ implies $\{x, x 1, \ldots, x(i-1)\} \subseteq \operatorname{dom}(T)$ for any $x \in[m]^{*}$ and $i \in[m]$.

Higher-Order Recursion Schemes. A higher-order recursion scheme [20] is a tree grammar for generating an infinite tree, where non-terminal symbols can take parameters. To preclude illegal parameters, each non-terminal symbol has a sort. The set of sorts is given by: $\kappa::=\circ \mid \kappa_{1} \rightarrow \kappa_{2}$. Intuitively, the sort o describes trees, and the sort $\kappa_{1} \rightarrow \kappa_{2}$ describes functions that take an element of sort $\kappa_{1}$ as input, and return an element of sort $\kappa_{2}$. The arity and order are defined by:

$$
\begin{aligned}
& \operatorname{arity}(\mathrm{o})=0 \quad \operatorname{arity}\left(\kappa_{1} \rightarrow \kappa_{2}\right)=1+\operatorname{arity}\left(\kappa_{2}\right) \\
& \operatorname{order}(\mathrm{o})=0 \quad \operatorname{order}\left(\kappa_{1} \rightarrow \kappa_{2}\right)=\max \left(1+\operatorname{order}\left(\kappa_{1}\right), \kappa_{2}\right)
\end{aligned}
$$

Formally, a higher-order recursion scheme (recursion scheme, for short) is a quadruple $\mathcal{G}=(\Sigma, \mathcal{N}, \mathcal{R}, S)$, where $\Sigma$ is a set of symbols called terminals and $\mathcal{N}$ is a set of symbols called non-terminals. Each terminal or non-terminal $\alpha$ has an associated sort, denoted by $\operatorname{sort}(\alpha)$. The order of the sort of a terminal must be 0 or 1 . We write $\operatorname{arity}(\alpha)$ for $\operatorname{arity}(\operatorname{sort}(\alpha))$ and call it the arity of $\alpha$. (Thus, $\Sigma$ is a ranked alphabet.) $\mathcal{R}$, called rewriting rules, is a finite map from $\mathcal{N}$ to $\lambda$-terms of the form $\lambda \widetilde{x}$.t where $t$ is an applicative term constructed from the variables $\widetilde{x}$, terminals, and non-terminals. $\mathcal{R}(F)$ must have sort $\operatorname{sort}(F)$ (under the standard simple type system). $S$ is a special non-terminal called the start symbol. The order of a recursion scheme $\mathcal{G}$ is the largest order of the sorts of its non-terminals. We use lower letters for terminals, and upper letters for non-terminals.

Given a recursion scheme $\mathcal{G}$, the rewriting relation $\longrightarrow_{\mathcal{G}}$ is the least relation that satisfies: (i) $F u_{1} \ldots, u_{k} \longrightarrow \mathcal{G}\left[u_{1} / x_{1}, \ldots, u_{k} / x_{k}\right] t$ if $\mathcal{R}(F)=\lambda x_{1} . \cdots \lambda x_{k} . t$, and (ii) $a t_{1} \cdots t_{n} \longrightarrow \mathcal{G} a t_{1} \cdots t_{i-1} t_{i}^{\prime} t_{i+1} \cdots t_{n}$ if $t_{i} \longrightarrow \mathcal{G} t_{i}^{\prime}$ 出 The value tree of $\mathcal{G}$, written $\llbracket \mathcal{G} \rrbracket$, is the (possibly infinite) $(\Sigma \cup\{\perp\})$-labelled tree generated by a fair, maximal reduction sequence from $S$. More precisely, define $t^{\perp}$ by $\left(a t_{1} \cdots t_{k}\right)^{\perp}=a t_{1} \perp \cdots t_{k}{ }^{\perp}$, and $\left(F t_{1} \cdots t_{k}\right)^{\perp}=\perp . \llbracket \mathcal{G} \rrbracket$ is $\bigsqcup\left\{t^{\perp} \mid S \longrightarrow_{\mathcal{G}}^{*} t\right\}$, where $\bigsqcup$ is the least upper bound with respect to the least compatible (i.e. closed under contexts) relation $\sqsubseteq$ on trees that satisfies $\perp \sqsubseteq T$ for every tree $T$.

Example 1. Consider the recursion scheme $\mathcal{G}_{0}=(\{\mathrm{a}, \mathrm{b}, \mathrm{c}\},\{S, F\}, \mathcal{R}, S)$, where $\mathcal{R}$ and the sorts of symbols are given by:

$$
\begin{aligned}
& \mathcal{R}=\{S \mapsto F \mathrm{bc}, \quad F \mapsto \lambda f \cdot \lambda x \cdot(\mathrm{a}(f x)(F f(f x)))\} \\
& \mathrm{a}: \mathrm{o} \rightarrow \mathrm{o} \rightarrow \mathrm{o}, \mathrm{b}: \mathrm{o} \rightarrow \mathrm{o}, \mathrm{c}: \mathrm{o}, S: \mathrm{o}, F:(\mathrm{o} \rightarrow \mathrm{o}) \rightarrow \mathrm{o} \rightarrow \mathrm{o}
\end{aligned}
$$

$\overline{2}$ Note that we allow only reductions of outermost redexes. 


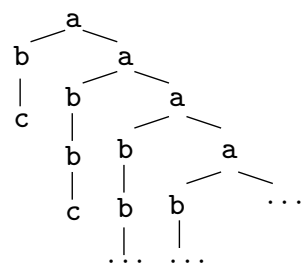

(a)

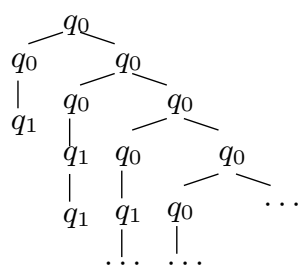

(b)

Fig. 1. The tree generated by the recursion scheme $\mathcal{G}_{0}$ and a run tree of it

$S$ is rewritten as follows, and the tree in Figure 1(a) is generated.

$$
S \longrightarrow F \mathrm{bc} \rightarrow \mathrm{a}(\mathrm{bc})(F \mathrm{~b}(\mathrm{bc})) \rightarrow \mathrm{a}(\mathrm{bc})(\mathrm{a}(\mathrm{b}(\mathrm{bcc}))(F \mathrm{~b}(\mathrm{~b}(\mathrm{bc}))) \rightarrow \cdots
$$

Trivial Automata Model Checking. The aim of model-checking a higher-order recursion scheme is to check whether the tree generated by the recursion scheme satisfies a certain regular property. In the present paper, we consider the properties described by trivial automata [2, which are sufficient for program verification problems considered in 1218 .

A trivial automaton $\mathcal{B}$ is a quadruple $\left(\Sigma, Q, \Delta, q_{0}\right)$, where $\Sigma$ is a set of input symbols, $Q$ is a finite set of states, $\Delta \subseteq Q \times \Sigma \times Q^{*}$ is a transition function, and $q_{0}$ is the initial state. A $\Sigma$-labeled tree $T$ is accepted by $\mathcal{B}$ if there is a $Q$-labeled tree $R$ (called a run tree) such that: (i) $\operatorname{dom}(T)=\operatorname{dom}(R)$; (ii) $R(\epsilon)=q_{0}$; and (iii) for every $x \in \operatorname{dom}(R),(R(x), T(x), R(x 1) \cdots R(x m)) \in \Delta$ where $m=\operatorname{arity}(T(x))$. For a trivial automaton $\mathcal{B}=\left(\Sigma, Q, \Delta, q_{0}\right)$ (with $\perp \notin \Sigma$ ), we write $\mathcal{B}^{\perp}$ for the trivial automaton $\left(\Sigma \cup\{\perp\}, Q, \Delta \cup\{(q, \perp, \epsilon) \mid q \in Q\}, q_{0}\right)$.

The trivial automata model checking is the problem of deciding whether $\llbracket \mathcal{G} \rrbracket$ is accepted by $\mathcal{B}^{\perp}$, given a recursion scheme $\mathcal{G}$ and a trivial automaton $\mathcal{B} 3$

Example 2. Consider the trivial automaton $\mathcal{B}_{0}=\left(\{\mathrm{a}, \mathrm{b}, \mathrm{c}\},\left\{q_{0}, q_{1}\right\}, \Delta, q_{0}\right)$, where $\Delta=\left\{\left(q_{0}, \mathrm{a}, q_{0} q_{0}\right),\left(q_{0}, \mathrm{~b}, q_{1}\right),\left(q_{1}, \mathrm{~b}, q_{1}\right),\left(q_{0}, \mathrm{c}, \epsilon\right),\left(q_{1}, \mathrm{c}, \epsilon\right)\right\} . \mathcal{B}_{0}$ accepts a $\{\mathrm{a}, \mathrm{b}, \mathrm{c}\}-$ labeled ranked tree just if a does not occur below b. The tree generated by $\mathcal{G}_{0}$ of Example 1 is accepted by $\mathcal{B}_{0}$. The run tree is shown in Figure 1(b).

Type Systems Equivalent to Trivial Automata Model Checking. One can construct an intersection type system (parameterized by a trivial automaton $\mathcal{B}=$ $\left.\left(\Sigma, Q, \Delta, q_{0}\right)\right)$ that is equivalent to trivial automata model checking, in the sense that a recursion scheme $\mathcal{G}$ is well typed if, and only if, $\llbracket \mathcal{G} \rrbracket$ is accepted by $\mathcal{B}^{\perp} \llbracket 12$. Define the set of types by:

$$
\theta \text { (atomic types) }::=q \mid \tau \rightarrow \theta \quad \tau \text { (intersections) }::=\bigwedge\left\{\theta_{1}, \ldots, \theta_{m}\right\}
$$

Here, $q$ ranges over the states of $\mathcal{B}$. We often write $\theta_{1} \wedge \cdots \wedge \theta_{m}$ or $\bigwedge_{i \in\{1, \ldots, m\}} \theta_{i}$ for $\bigwedge\left\{\theta_{1}, \ldots, \theta_{m}\right\}$. We also write $\top$ for $\bigwedge \emptyset$, and $\theta$ for $\bigwedge\{\theta\} . \wedge$ binds tighter

${ }^{3}$ In the literature 2016, it is often assumed that the value tree of $\mathcal{G}$ does not contain $\perp$. Under that assumption, the acceptance by $\mathcal{B}^{\perp}$ and $\mathcal{B}$ are equivalent. 
than $\rightarrow$. Intuitively, $q$ is a refinement of sort $\circ$, describing trees accepted by $\mathcal{B}$ with the initial state replaced by $q . \theta_{1} \wedge \cdots \wedge \theta_{m} \rightarrow \theta$ describes functions that take an element that has types $\theta_{1}, \ldots, \theta_{m}$ as input, and return an element of type $\theta$. For example, $q_{0} \wedge q_{1} \rightarrow q_{0}$ describes a function that takes a tree that can be accepted from both $q_{0}$ and $q_{1}$, and returns a tree accepted from $q_{0}$. We define the refinement relation $\theta:: \kappa$ inductively by: (i) $q::$ o for every $q \in Q$ and (ii) $\left(\bigwedge_{i \in S} \theta_{i} \rightarrow \theta\right)::\left(\kappa_{1} \rightarrow \kappa_{2}\right)$ if $\forall i \in S . \theta_{i}:: \kappa_{1}$ and $\theta:: \kappa_{2}$.

The typing rules for terms and rewriting rules are given as follows.

$$
\begin{array}{ccr}
\frac{\left(q, a, q_{1} \cdots q_{k}\right) \in \Delta}{\Gamma \vdash^{\mathcal{B}} a: q_{1} \rightarrow \cdots q_{k} \rightarrow q} & \frac{x: \theta \in \Gamma}{\Gamma \vdash^{\mathcal{B}} x: \theta} & \frac{\forall i \in S .\left(\Gamma \vdash^{\mathcal{B}} t: \theta_{i}\right)}{\Gamma \vdash^{\mathcal{B}} t: \bigwedge_{i \in S} \theta_{i}} \\
\Gamma \vdash^{\mathcal{B}} t_{1}: \tau \rightarrow \theta & \Gamma, x: \theta_{1}, \ldots, x: \theta_{m} \vdash^{\mathcal{B}} t: \theta & \operatorname{dom}(\Gamma) \subseteq \operatorname{dom}(\mathcal{R}) \\
\frac{\Gamma \vdash^{\mathcal{B}} t_{2}: \tau}{\Gamma \vdash^{\mathcal{B}} t_{1} t_{2}: \theta} & \frac{x \notin \operatorname{dom}(\Gamma)}{\Gamma \vdash^{\mathcal{B}} \lambda x . t: \theta_{1} \wedge \cdots \wedge \theta_{m} \rightarrow \theta} & \frac{\forall(F: \theta) \in \Gamma \cdot\left(\Gamma \vdash^{\mathcal{B}} \mathcal{R}(F): \theta\right)}{\vdash^{\mathcal{B}} \mathcal{R}: \Gamma}
\end{array}
$$

Here, $\Gamma$ is a set of bindings of the form $x: \theta$ where non-terminals are also treated as variables, and $\Gamma$ may contain more than one binding for each variable. We write $\operatorname{dom}(\Gamma)$ for $\{x \mid x: \theta \in S\}$. A recursion scheme $\mathcal{G}=(\Sigma, \mathcal{N}, \mathcal{R}, S)$ is well typed under $\Gamma$, written $\vdash^{\mathcal{B}} \mathcal{G}: \Gamma$, if $\vdash^{\mathcal{B}} \mathcal{R}: \Gamma, \forall(F: \theta) \in \Gamma$. $(\theta:: \operatorname{sort}(F))$, and $S: q_{0} \in \Gamma$. We write $\vdash^{\mathcal{B}} \mathcal{G}$ if $\vdash^{\mathcal{B}} \mathcal{G}: \Gamma$ for some $\Gamma$.

The following theorem guarantees the correspondence between model checking and type checking.

Theorem 1 (Kobayashi [12]). $\llbracket \mathcal{G} \rrbracket$ is accepted by $\mathcal{B}^{\perp}$ if and only if $\vdash^{\mathcal{B}} \mathcal{G}$.

Example 3. Recall the recursion scheme $\mathcal{G}_{0}$ in Example1 1 and the trivial automaton $\mathcal{B}_{0}$ in Example 2. $\vdash^{\mathcal{B}_{0}} \mathcal{G}_{0}: \Gamma$ holds for $\Gamma=\left\{S: q_{0}, F:\left(q_{1} \rightarrow q_{1}\right) \wedge\left(q_{1} \rightarrow\right.\right.$ $\left.\left.q_{0}\right) \rightarrow q_{1} \rightarrow q_{0}\right\}$

Theorem 1 above yields a straightforward, fixedpoint-based model checking algorithm. Let Shrink $_{\mathcal{G}, \mathcal{B}}$ be the function on type environments defined by: $\operatorname{Shrink}_{\mathcal{G}, \mathcal{B}}(\Gamma)=\left\{F: \theta \in \Gamma \mid \Gamma \vdash^{\mathcal{B}} \mathcal{R}(F): \theta\right\}$, and let $\Gamma_{\max }$ be $\{F: \theta \mid$ $F \in \mathcal{N}, \theta:: \operatorname{sort}(F)\}$. Then, by the definition, $\vdash^{\mathcal{B}} \mathcal{G}: \Gamma$ if and only if there exists $\Gamma \subseteq \Gamma_{\max }$ such that $\Gamma \subseteq \operatorname{Shrink}_{\mathcal{G}, \mathcal{B}}(\Gamma)$ and $S: q_{0} \in \Gamma$. (Note that $\Gamma \subseteq \operatorname{Shrink}_{\mathcal{G}, \mathcal{B}}(\Gamma)$ if and only if $\vdash^{\mathcal{B}} \mathcal{R}: \Gamma$.) Thus, to check whether $\vdash^{\mathcal{B}} \mathcal{G}$ holds, it is sufficient to compute the greatest fixedpoint $\Gamma_{\mathbf{g f p}}$ of $\mathbf{S h r i n k}_{\mathcal{G}, \mathcal{B}}$ and checks whether $S: q_{0} \in \Gamma_{\text {gfp }}$. This is Kobayashi's naïve algorithm.

NAIVE ALGORITHM [12] :

1. $\Gamma:=\Gamma_{\max }$

2. Repeat $\Gamma:=\operatorname{Shrink}_{\mathcal{G}, \mathcal{B}}(\Gamma)$ until $\Gamma=\operatorname{Shrink}_{\mathcal{G}, \mathcal{B}}(\Gamma)$;

3. Output whether $S: q_{0} \in \Gamma$.

Suppose that the size of $\mathcal{B}$ and the largest size of sorts of symbols are fixed. Then, the size of $\Gamma_{\max }$ is linear in the size of $\mathcal{G}$, since for a given $\kappa$, the number of types that satisfy $\theta:: \kappa$ is bounded above by a constant. Thus, using Rehof and Mogensen's optimization [22, the above algorithm is made linear in the size 
of $\mathcal{G}$. The algorithm does not work in practice, however, as the constant factor is too large. Even at the first iteration of the fixedpoint computation, we need to pick each binding $F: \theta$ from $\Gamma_{\max }$ and check whether $\Gamma_{\max } \vdash^{\mathcal{B}} \mathcal{R}(F): \theta$ holds. This is impractical, as $\Gamma_{\max }$ is too large; In fact, even when $|Q|=2$, for a symbol of sort $((\mathrm{o} \rightarrow \mathrm{o}) \rightarrow \mathrm{o}) \rightarrow \mathrm{o}$, the number of corresponding types is $2^{513} \approx 10^{154}$.

Kobayashi's hybrid algorithm [10] starts the greatest fixedpoint computation from a type environment much smaller than $\Gamma_{\mathbf{m a x}}$. To find an appropriate startpoint of the fixedpoint computation, his algorithm reduces the recursion scheme a finite number of times, and infers candidates of the types of each non-terminal, by observing how each non-terminal is used in the reduction sequence. The following is an outline of the hybrid algorithm (see [10] for more details):

HYBRID ALGORITHM [10] :

1. Reduce $S$ a finite number of steps;

2. If a property violation is found, output 'no' and halt;

3. $\Gamma:=$ type bindings extracted from the reduction sequence;

4. Repeat $\Gamma:=\operatorname{Shrink}_{\mathcal{G}, \mathcal{B}}(\Gamma)$ until $\Gamma=\operatorname{Shrink}_{\mathcal{G}, \mathcal{B}}(\Gamma)$;

5. If $S: q_{0} \in \Gamma$ then output 'yes' and halt;

6 . Go back to 1 and reduce $S$ further.

The algorithm works well in practice [10 18, but has some limitations: (i) No theoretical guarantee that the algorithm is efficient. In fact, the worst-case running time is hyper-exponential [13]: see Section 5. (ii) The efficiency of the algorithm crucially depends on the selection of terms to be reduced in Step 1 . The implementation relies on heuristics for choosing reduced terms, and there is no theoretical justification for it. (iii) It works only for deterministic trivial automata (i.e. trivial automata such that $\left|\Delta \cap\{q\} \times\{a\} \times Q^{*}\right| \leq 1$ for every $q \in Q, a \in \Sigma$.) Though it is possible to extend the algorithm to remove the restriction, it is unclear whether the resulting algorithm is efficient in practice.

The limitations above motivated us to look for yet another algorithm, which is efficient both in practice and in theory (where an important criterion for the latter is that the time complexity should be linear in the size of the recursion scheme, under the assumption that the other parameters are fixed). That is the subject of this paper, discussed in the following sections.

\section{The New Model Checking Algorithm}

\subsection{Main Idea}

In the previous section, a reader may have wondered why we do not compute the least fixedpoint, instead of the greatest one. The naïve least fixedpoint computation however does not work. Let us define $\mathcal{F}_{\mathcal{B}}$ by: $\mathcal{F}_{\mathcal{B}}(\Gamma)=\left\{F: \theta \mid \Gamma \vdash^{\mathcal{B}} \mathcal{R}(F)\right.$ : $\theta$ \}. How about computing the least fixedpoint $\Gamma_{\text {lfp }}$ of $\mathcal{F}_{\mathcal{B}}$, and checking whether $S: q_{0} \in \Gamma_{\mathbf{l f p}}$ ? This does not work for two reasons. First of all, $S: q_{0} \in \Gamma_{\mathbf{l f p}}$ is not a necessary condition for the well-typedness of $\mathcal{G}$. For example, for $\mathcal{G}_{0}$ of Example 1. $\Gamma_{\text {lfp }}=\emptyset$. Secondly, for each iteration to compute $\mathcal{F}_{\mathcal{B}}(\emptyset), \mathcal{F}_{\mathcal{B}}^{2}(\emptyset), \mathcal{F}_{\mathcal{B}}^{3}(\emptyset), \ldots$, 
we have to guess a type $\theta$ of $F$ and check whether $\Gamma \vdash^{\mathcal{B}} \mathcal{R}(F): \theta$. The possible types of $F$ are however too many, hence the same problem as the greatest fixedpoint computation.

The discussion above however suggests that a least fixedpoint computation may work if, in $\Gamma \vdash^{\mathcal{B}} \mathcal{R}(F): \theta$, (i) we relax the condition on $\Gamma$ (that $\Gamma$ must have been obtained from the previous iteration steps), and (ii) we impose a restriction on $\theta$, to disallow $\theta$ to be synthesized "out of thin air". This observation motivates us to modify $\mathcal{F}_{\mathcal{B}}(\Gamma)$ as follows:

$$
\mathcal{F}_{\mathcal{B}}^{\prime}(\Gamma)=\bigcup\left\{\left\{F: \theta^{\prime}\right\} \cup \Gamma^{\prime} \mid \Gamma^{\prime} \vdash^{\mathcal{B}} \mathcal{R}(F): \theta^{\prime}, \Gamma \preceq_{O} \Gamma^{\prime}, \theta \preceq_{P} \theta^{\prime},(F: \theta) \in \Gamma\right\} .
$$

Here, $\Gamma \preceq_{O} \Gamma^{\prime}$ (which will be defined later) indicates that $\Gamma^{\prime}$ is not identical, but somehow similar to $\Gamma$. The condition " $\theta \preceq_{P} \theta^{\prime}$ for some $F: \theta \in \Gamma$ " also indicates that $F: \theta^{\prime}$ is somehow similar to an existing type binding $F: \theta$ of $\Gamma$.

To see how $\preceq_{O}$ and $\preceq_{P}$ may be defined, let us consider $\mathcal{G}_{0}$ and $\mathcal{B}_{0}$ of Examples 1 and 2 , In order for $\llbracket \mathcal{G}_{0} \rrbracket$ to be accepted by $\mathcal{B}_{0}{ }^{\perp}, S$ should have type $q_{0}$. So, let us first put $S: q_{0}$ into the initial type environment: $\Gamma_{0}:=\left\{S: q_{0}\right\}$.

Now, in order for the body $F \mathrm{bc}$ of $S$ to have type $q_{0}, F$ must have a type of the form $\cdots \rightarrow \cdots \rightarrow q_{0}$. So, let us put $F: \top \rightarrow \top \rightarrow q_{0}: \Gamma_{1}:=\left\{S: q_{0}, F: \top \rightarrow\right.$ $\left.\top \rightarrow q_{0}\right\}$.

Let us now look at the definition of $F$, to check whether the body of $F$ has type $\top \rightarrow \top \rightarrow q_{0}$. The body doesn't, but it has a slightly modified type: $(\top \rightarrow$ $\left.q_{0}\right) \rightarrow \top \rightarrow q_{0}$, so we update the type of $F: \Gamma_{2}:=\left\{S: q_{0}, F:\left(\top \rightarrow q_{0}\right) \rightarrow \top \rightarrow q_{0}\right\}$.

Going back to the definition of $S$, we know that $F$ is required to have a type like $\left(q_{1} \rightarrow q_{0}\right) \rightarrow \top \rightarrow q_{0}$ (because $\mathrm{b}$ has type $q_{1} \rightarrow q_{0}$, not $\left.\top \rightarrow q_{0}\right)$. Thus, we further update the type of $F: \Gamma_{3}:=\left\{S: q_{0}, F:\left(q_{1} \rightarrow q_{0}\right) \rightarrow \top \rightarrow q_{0}\right\}$.

By looking at the definition of $F$ again, we know that $x$ should have type $q_{1}$ and that $f$ should have $q_{1}$ as a return type, from the first and second arguments of a respectively. Thus, we get an updated type environment: $\Gamma_{4}:=\left\{S: q_{0}, F:\left(q_{1} \rightarrow\right.\right.$ $\left.\left.q_{0}\right) \wedge\left(\top \rightarrow q_{1}\right) \rightarrow q_{1} \rightarrow q_{0}\right\}$. By checking the definition of $S$ again, we get:

$$
\Gamma_{5}:=\left\{S: q_{0}, F:\left(q_{1} \rightarrow q_{0}\right) \wedge\left(q_{1} \rightarrow q_{1}\right) \rightarrow q_{1} \rightarrow q_{0}\right\} .
$$

Thus, we have obtained enough type information for $\mathcal{G}_{0}$ (recall Example 3).

In the above example, the type of $F$ has been expanded as follows.

$$
\begin{aligned}
& \top \preceq_{O} \top \rightarrow \top \rightarrow q_{0} \preceq_{P}\left(\top \rightarrow q_{0}\right) \rightarrow \top \rightarrow q_{0} \preceq_{O}\left(q_{1} \rightarrow q_{0}\right) \rightarrow \top \rightarrow q_{0} \\
& \preceq_{P}\left(q_{1} \rightarrow q_{0}\right) \wedge\left(\top \rightarrow q_{1}\right) \rightarrow q_{1} \rightarrow q_{0} \preceq_{O}\left(q_{1} \rightarrow q_{0}\right) \wedge\left(q_{1} \rightarrow q_{1}\right) \rightarrow q_{1} \rightarrow q_{0} .
\end{aligned}
$$

Here, the expansions represented by $\preceq_{O}$ come from constraints on call sites of $F$, and those represented by $\preceq_{P}$ come from constraints on the definition of $F$. We shall formally define these expansion relations and obtain a fixedpoint-based model checking algorithm in the following sections.

Remark 1. A reader familiar with game semantics [120] may find a connection between the type expansion sequence above and a play of a function. For example, the type $\left(\top \rightarrow q_{0}\right) \rightarrow \top \rightarrow q_{0}$ may be considered an abstraction of a state of 
a play where the opponent of $F$ has requested a tree of type $q_{0}$, and the proponent has requested a tree of type $q_{0}$ in response. The type $\left(q_{1} \rightarrow q_{0}\right) \rightarrow \top \rightarrow q_{0}$ represents the next state, where the opponent has requested a tree of type $q_{1}$ in response, and the type $\left(q_{1} \rightarrow q_{0}\right) \wedge\left(\top \rightarrow q_{1}\right) \rightarrow q_{1} \rightarrow q_{0}$ represents the state where, in response to it, the proponent has requested trees of type $q_{1}$ to the first and second arguments. Thus, the expansion relations $\preceq_{O}$ and $\preceq_{P}$ represent opponent's and proponent's moves respectively. Although we do not make this connection formal, this game semantic intuition may help understand how our algorithm described below works. In particular, the intuition helps us understand why necessary type information can be obtained by gradually expanding types as in the example above; for a valid type of a function 4 there should be a corresponding play of the function, and by following the play, one can obtain a type expansion sequence that leads to the valid type.

\subsection{Expansion Relations}

We now formally define the expansion relations $\preceq_{O}$ and $\preceq_{P}$ mentioned above. They are inductively defined by the following rules.

$$
\begin{aligned}
& \overline{q \preceq O q} \quad \frac{}{q \preceq_{P} q} \quad \frac{\tau \preceq_{P} \tau^{\prime} \quad \theta \preceq_{O} \theta^{\prime}}{\tau \longrightarrow \theta \preceq_{O} \tau^{\prime} \rightarrow \theta^{\prime}} \quad \frac{\tau \preceq_{O} \tau^{\prime} \quad \theta \preceq_{P} \theta^{\prime}}{\tau \longrightarrow \theta \preceq_{P} \tau^{\prime} \rightarrow^{\prime}} \\
& \forall j \in S^{\prime} \backslash S . \exists q . \theta_{j}^{\prime}=\top \rightarrow \cdots \rightarrow \top \rightarrow q \\
& \frac{\forall j \in S . \theta_{j} \preceq_{O} \theta_{j}^{\prime} \quad S \subseteq S^{\prime}}{\bigwedge_{j \in S} \theta_{j} \preceq_{O} \bigwedge_{j \in S^{\prime}} \theta_{j}^{\prime}} \quad \frac{\forall j \in S . \theta_{j} \preceq_{P} \theta_{j}^{\prime}}{\bigwedge_{j \in S} \theta_{j} \preceq_{P} \bigwedge_{j \in S} \theta_{j}^{\prime}}
\end{aligned}
$$

Note that the four relations: $\theta \preceq_{O} \theta^{\prime}, \tau \preceq_{O} \tau^{\prime}, \theta \preceq_{P} \theta^{\prime}$, and $\tau \preceq_{P} \tau^{\prime}$ are defined simultaneously. Notice also that $\preceq_{P}$ and $\preceq_{O}$ are swapped in the argument position of arrow types; this is analogous to the contravariance of the standard subtyping relation in the argument position of function types. Another way to understand the relations is: $\theta \preceq_{O} \theta^{\prime}\left(\theta \preceq_{P} \theta^{\prime}\right.$, resp.) holds if $\theta^{\prime}$ is obtained from $\theta$ by adding atomic types (of the form $q$ ) to positive (negative, resp.) positions.

In the last two rules, $S$ can be an empty set. So, we can derive $\top \preceq_{O} \top \rightarrow q_{0}$, from which $\top \rightarrow \top \rightarrow q_{0} \preceq_{P}\left(\top \rightarrow q_{0}\right) \rightarrow \top \rightarrow q_{0}$ follows. The expansion relation $\preceq_{O}$ is extended to type environments by: $\Gamma \preceq_{O} \Gamma^{\prime}$ if and only if $\forall x \in$ $\left.\operatorname{dom}(\Gamma) \cup \operatorname{dom}\left(\Gamma^{\prime}\right) \cdot \Gamma(x) \preceq_{O} \Gamma^{\prime}(x)\right)$. Here, $\Gamma(x)=\bigwedge\{\theta \mid x: \theta \in \Gamma\} . \Gamma \preceq_{P} \Gamma^{\prime}$ is defined in a similar manner.

Let $\leq$ be the standard subtyping relation on intersection types. Then, $\theta_{2} \preceq O$ $\theta_{1}$ or $\theta_{1} \preceq_{P} \theta_{2}$ imply $\theta_{1} \leq \theta_{2}$, but not vice versa. For example, $\top \rightarrow q_{0} \leq\left(q_{1} \rightarrow\right.$ $\left.q_{0}\right) \rightarrow q_{0}$ but $\top \rightarrow q_{0} \npreceq_{P}\left(q_{1} \rightarrow q_{0}\right) \rightarrow q_{0}$

\subsection{Type Generation Rules}

We now define the relation $\Gamma_{1} \triangleright \Gamma_{2} \vdash{ }_{P}^{\mathcal{B}} t: \theta_{1} \triangleright \theta_{2}$, which means that, given a candidate of type judgment $\Gamma_{1} \vdash t$ : $\theta_{1}$ (which may not be valid), a valid

\footnotetext{
${ }^{4}$ Strictly speaking, we should interpret a type as a kind of linear type; for example, the type $q_{1} \rightarrow q_{0}$ should be interpreted as a function that takes a tree of type $q_{1}$ and uses it at least once to return a tree of type $q_{0}$.
} 
judgment $\Gamma_{2} \vdash^{\mathcal{B}} t: \theta_{2}$ is obtained by "adjusting" $\Gamma_{1}$ and $\theta_{1}$ in a certain manner. $\Gamma_{1} \triangleright \Gamma_{2} \vdash_{P}^{\mathcal{B}} t: \theta_{1} \triangleright \theta_{2}$ corresponds to the condition $\left(\Gamma_{2} \vdash^{\mathcal{B}} t: \theta_{2}\right) \wedge\left(\Gamma_{1} \preceq O\right.$ $\left.\Gamma_{2}\right) \wedge\left(\theta_{1} \preceq_{P} \theta_{2}\right)$ used in the informal definition of $\mathcal{F}_{\mathcal{B}}^{\prime}$ in Section 3.1. In fact, the rules below ensure that $\Gamma_{1} \triangleright \Gamma_{2} \vdash_{P}^{\mathcal{B}} t: \theta_{1} \triangleright \theta_{2}$ implies that $\left(\Gamma_{2} \vdash^{\mathcal{B}} t: \theta_{2}\right) \wedge\left(\Gamma_{1} \preceq O\right.$ $\left.\Gamma_{2}\right) \wedge\left(\theta_{1} \preceq_{P} \theta_{2}\right)$ holds. Thus, the "adjustment" of $\Gamma_{1}$ and $\theta_{1}$ is allowed only in a restricted manner. For example, $\left(f: q_{1} \rightarrow q_{0}\right) \triangleright\left(f: q_{1} \rightarrow q_{0}, x: q_{1}\right) \vdash_{P}^{\mathcal{B}} f x: q_{0} \triangleright q_{0}$ is allowed, but $\left(f: \top \rightarrow q_{0}\right) \triangleright\left(f: q_{1} \rightarrow q_{0}, x: q_{1}\right) \vdash_{P}^{\mathcal{B}} f x: q_{0} \triangleright q_{0}$ is not. In the latter, the change of the type of function $f$ makes the assumption on the behavior of $f$ that upon receiving a request for tree of type $q_{0}, f$ requests a tree of type $q_{1}$ as an argument. That assumption is speculative and should be avoided, as its validity can be determined only by looking at the environment, not the term $f x$.

Definition 1. $\Gamma_{1} \triangleright \Gamma_{2} \vdash_{P}^{\mathcal{B}} t: \theta_{1} \triangleright \theta_{2}$ and $\Gamma_{1} \triangleright \Gamma_{2} \vdash_{P}^{\mathcal{B}} t: \tau_{1} \triangleright \tau_{2}$ are the least relations that satisfy the following rules.

$$
\begin{gathered}
\frac{\theta_{1} \preceq_{P} \theta_{2}}{x: \theta_{2} \triangleright x: \theta_{2} \vdash_{P}^{\mathcal{B}} x: \theta_{1} \triangleright \theta_{2}}(\mathrm{VARP}) \frac{\tau_{1} \preceq_{O} \theta_{2}}{x: \tau_{1} \triangleright x: \theta_{2} \vdash_{P}^{\mathcal{B}} x: \theta_{2} \triangleright \theta_{2}} \\
\frac{\left(q, a, q_{1} \cdots q_{n}\right) \in \Delta \quad \forall i \in\{1, \ldots, n\} . \tau_{i} \in\left\{\top, q_{i}\right\}}{\emptyset \triangleright \emptyset \vdash_{P}^{\mathcal{B}} a:\left(\tau_{1} \rightarrow \cdots \rightarrow \tau_{n} \rightarrow q\right) \triangleright\left(q_{1} \rightarrow \cdots \rightarrow q_{n} \rightarrow q\right)} \\
\frac{\Gamma_{1} \triangleright \Gamma_{1}^{\prime} \vdash_{P}^{\mathcal{B}} t_{1}:(\tau \rightarrow \theta) \triangleright\left(\tau^{\prime} \rightarrow \theta^{\prime}\right) \quad \Gamma_{2} \triangleright \Gamma_{2}^{\prime} \vdash_{P}^{\mathcal{B}} t_{2}: \tau^{\prime \prime} \triangleright \tau^{\prime}}{\Gamma_{1} \cup \Gamma_{2} \triangleright \Gamma_{1}^{\prime} \cup \Gamma_{2}^{\prime} \vdash_{P}^{\mathcal{B}} t_{1} t_{2}: \theta \triangleright \theta^{\prime}} \\
\frac{\left(\Gamma_{1}, x: \tau_{1}\right) \triangleright\left(\Gamma_{2}, x: \tau_{2}\right) \vdash_{P}^{\mathcal{B}} t: \theta_{1} \triangleright \theta_{2}}{\Gamma_{1} \triangleright \Gamma_{2} \vdash{ }_{P}^{\mathcal{B}} \lambda x . t:\left(\tau_{1} \rightarrow \theta_{1}\right) \triangleright\left(\tau_{2} \rightarrow \theta_{2}\right)} \\
\forall i \in S .\left(\Gamma_{i} \triangleright \Gamma_{i}^{\prime} \vdash{ }_{P}^{\mathcal{B}} t: \theta_{i} \triangleright \theta_{i}^{\prime}\right) \\
\frac{\left(\bigcup_{i \in S} \Gamma_{i} \triangleright{ }_{i \in S} \Gamma_{i}^{\prime}\right) \vdash_{P}^{\mathcal{B}} t:\left(\bigwedge_{i \in S} \cdot \theta_{i} \triangleright\left(\bigwedge_{i \in S} \cdot \theta_{i}^{\prime}\right)\right.}{}
\end{gathered}
$$

In the rules above, we write $x: \bigwedge_{i \in S} \theta_{i}$ for $\left\{x: \theta_{i} \mid i \in S\right\}$. It is implicitly assumed that the sort of each variable is respected, i.e., if $x: \theta \in \Gamma$, then $\theta:: \operatorname{sort}(x)$ must hold. The rule VARP is used for adjusting the type of $x$ to the type provided by the environment, while the rule $\mathrm{VARO}$ is used for adjusting the environment to the type of $x$. For example, $\left(x: q_{1} \rightarrow q_{2}\right) \triangleright\left(x: q_{1} \rightarrow q_{2}\right) \vdash_{P}^{\mathcal{B}} x:\left(\top \rightarrow q_{2}\right) \triangleright\left(q_{1} \rightarrow q_{2}\right)$ is obtained from the former, and $x: \top \triangleright\left(x: \top \rightarrow q_{2}\right) \vdash_{P}^{\mathcal{B}} x:\left(\top \rightarrow q_{2}\right) \triangleright\left(\top \rightarrow q_{2}\right)$ is obtained from the latter. In the rule APP, the left and right premises adjust the types of the function and the argument respectively. (In the game semantic view, the former accounts for a move of the function, and the latter accounts for a move of the argument.)

\subsection{Model Checking Algorithm}

We are now ready to describe the new algorithm. Let $\mathcal{G}=(\Sigma, \mathcal{N}, \mathcal{R}, S)$ be a recursion scheme and $\mathcal{B}$ be a trivial automaton. Define $\operatorname{Expand}_{\mathcal{G}, \mathcal{B}}$ by: 
$\operatorname{Expand}_{\mathcal{G}, \mathcal{B}}(\Gamma)=\Gamma \cup\left(\bigcup\left\{\Gamma^{\prime} \cup\left\{F: \theta^{\prime}\right\} \mid \Gamma_{1} \triangleright \Gamma^{\prime} \vdash_{P}^{\mathcal{B}} \mathcal{R}(F): \theta \triangleright \theta^{\prime}, F: \theta \in \Gamma, \Gamma_{1} \subseteq \Gamma\right\}\right)$

Our new algorithm just consists of two fixedpoint computations:

NEW ALGORITHM :

1. $\Gamma:=\left\{S: q_{0}\right\}$;

2. Repeat $\Gamma:=\operatorname{Expand}_{\mathcal{G}, \mathcal{B}}(\Gamma)$ until $\Gamma=\operatorname{Expand}_{\mathcal{G}, \mathcal{B}}(\Gamma)$;

3. Repeat $\Gamma:=\operatorname{Shrink}_{\mathcal{G}, \mathcal{B}}(\Gamma)$ until $\Gamma=\operatorname{Shrink}_{\mathcal{G}, \mathcal{B}}(\Gamma)$;

4. Output whether $S: q_{0} \in \Gamma$.

We first expand the set of type candidates by using Expand $_{\mathcal{G}, \mathcal{B}}$, and then shrink it by filtering out invalid types by $\mathbf{S h r i n k}_{\mathcal{G}, \mathcal{B}}$. The only change from the naïve algorithm is that $\Gamma_{\mathbf{m a x}}$ has been replaced by the least fixedpoint of $\mathbf{E x p a n d}_{\mathcal{G}, \mathcal{B}}$.

Example 4. Recall $\mathcal{G}_{0}$ and $\mathcal{B}_{0}$ of Examples 1 and 2] Let $\Gamma_{0}=\left\{S: q_{0}\right\}$. We have:

$$
\begin{aligned}
& \Gamma_{1}=\operatorname{Expand}_{\mathcal{G}_{0}, \mathcal{B}_{0}}\left(\Gamma_{0}\right)=\left\{S: q_{0}, F: \top \rightarrow \top \rightarrow q_{0}\right\} \\
& \Gamma_{2}=\operatorname{Expand}_{\mathcal{G}_{0}, \mathcal{B}_{0}}\left(\Gamma_{1}\right)=\Gamma_{1} \cup\left\{F:\left(\top \rightarrow q_{0}\right) \rightarrow \top \rightarrow q_{0}\right\} \\
& \Gamma_{3}=\operatorname{Expand}_{\mathcal{G}_{0}, \mathcal{B}_{0}}\left(\Gamma_{2}\right)=\Gamma_{2} \cup\left\{F:\left(q_{1} \rightarrow q_{0}\right) \rightarrow \top \rightarrow q_{0}, \ldots\right\} \\
& \Gamma_{4}=\operatorname{Expand}_{\mathcal{G}_{0}, \mathcal{B}_{0}}\left(\Gamma_{3}\right)=\Gamma_{3} \cup\left\{F:\left(q_{1} \rightarrow q_{0}\right) \rightarrow q_{1} \rightarrow q_{0}, \ldots\right\} \\
& \Gamma_{5}=\operatorname{Expand}_{\mathcal{G}_{0}, \mathcal{B}_{0}}\left(\Gamma_{4}\right)=\Gamma_{3} \cup\left\{F:\left(q_{1} \rightarrow q_{0}\right) \wedge\left(\top \rightarrow q_{1}\right) \rightarrow q_{1} \rightarrow q_{0}, \ldots\right\} \\
& \Gamma_{6}=\operatorname{Expand}_{\mathcal{G}_{0}, \mathcal{B}_{0}}\left(\Gamma_{5}\right)=\Gamma_{4} \cup\left\{F:\left(q_{1} \rightarrow q_{0}\right) \wedge\left(q_{1} \rightarrow q_{1}\right) \rightarrow q_{1} \rightarrow q_{0}, \ldots\right\} \\
& \Gamma_{7}=\operatorname{Expand}_{\mathcal{G}_{0}, \mathcal{B}_{0}}\left(\Gamma_{6}\right)=\Gamma_{6}
\end{aligned}
$$

In the second step (for computing $\Gamma_{2}$ ), the type $\left(\top \rightarrow q_{0}\right) \rightarrow \top \rightarrow q_{0}$ of $F$ is obtained from the following derivation.

$$
\frac{\emptyset \triangleright f: \top \rightarrow q_{0} \vdash_{P}^{\mathcal{B}} \mathrm{a}(f x):\left(\top \rightarrow q_{0}\right) \triangleright\left(q_{0} \rightarrow q_{0}\right) \quad \Delta_{1} \triangleright \Delta_{1} \vdash_{P}^{\mathcal{B}}(F f(f x)): q_{0} \triangleright q_{0}}{\frac{\Delta_{1} \triangleright \Delta_{2} \vdash_{P}^{\mathcal{B}} \mathrm{a}(f x)(F f(f x)): q_{0} \triangleright q_{0}}{\Delta_{1} \triangleright \Delta_{1} \vdash_{P}^{\mathcal{B}} \lambda f . \lambda x . \mathrm{a}(f x)(F f(f x)): \top \rightarrow \top \rightarrow q_{0} \triangleright\left(\top \rightarrow q_{0}\right) \rightarrow \top \rightarrow q_{0}}}
$$

Here, $\Delta_{1}=F: \top \rightarrow \top \rightarrow q_{0}, \Delta_{2}=\Delta_{1} \cup\left\{f: \top \rightarrow q_{0}\right\}$, and $\emptyset \triangleright f: \top \rightarrow q_{0} \vdash{ }_{P}^{\mathcal{B}}$ $\mathrm{a}(f x):\left(\top \rightarrow q_{0}\right) \triangleright\left(q_{0} \rightarrow q_{0}\right)$ is derivable from $\emptyset \triangleright f:\left(\top \rightarrow q_{0}\right) \vdash{ }_{P}^{\mathcal{B}} f: \top \rightarrow q_{0} \triangleright$ $\top \rightarrow q_{0}$ and $\emptyset \triangleright \emptyset \vdash_{P}^{\mathcal{B}}$ a $:\left(\top \rightarrow \top \rightarrow q_{0}\right) \triangleright\left(q_{0} \rightarrow q_{0} \rightarrow q_{0}\right)$.

By repeatedly applying $\mathbf{S h r i n k}_{\mathcal{G}, \mathcal{B}}$ to $\Gamma_{6}$, we obtain: $\Gamma=\left\{S: q_{0}, F:\left(q_{1} \rightarrow\right.\right.$ $\left.\left.q_{0}\right) \wedge\left(q_{1} \rightarrow q_{1}\right) \rightarrow q_{1} \rightarrow q_{0}, F:\left(\top \rightarrow q_{0}\right) \rightarrow \top \rightarrow q_{0}, \ldots\right\}$ as a fixedpoint. Since $S: q_{0} \in \Gamma$, we know that $\mathcal{G}_{0}$ is well-typed, i.e. $\llbracket \mathcal{G} \rrbracket$ is accepted by $\mathcal{B}^{\perp}$.

The least fixedpoint $\Gamma_{6}$ of Expand $_{\mathcal{G}_{0}, \mathcal{B}_{0}}$ contains type bindings like $F:(\top \rightarrow$ $\left.q_{0}\right) \rightarrow \top \rightarrow q_{0}$, which are not required for typing $\mathcal{G}$ (recall Example 3 ). However, Expand $_{\mathcal{G}_{0}, \mathcal{B}_{0}}$ does not add completely irrelevant type bindings like $F: \top \rightarrow \top \rightarrow$ $q_{1}$. Thus, we can expect that the least fixedpoint of $\operatorname{Expand}_{\mathcal{G}_{0}, \mathcal{B}_{0}}$ is often much smaller than $\Gamma_{\mathbf{m a x}}$, which will be confirmed by the experiments in Section 5 .

\section{Correctness of the Algorithm}

This section discusses the correctness and complexity of the algorithm. 
Termination and Complexity. The termination follows immediately from the following facts: (i) $\Gamma$ increases monotonically in the first loop, (ii) $\Gamma$ decreases monotonically in the second loop, and (iii) $\Gamma$ ranges over a finite set.

Theorem 2. The algorithm always terminates and outputs "yes" or "no".

From the termination argument, the complexity result also follows.

Theorem 3. Suppose that both (i) the largest size of the sorts of non-terminals and terminals of $\mathcal{G}$ and $(i i)$ the size of automaton $\mathcal{B}$ are fixed. Then, the algorithm terminates in time quadratic in $|\mathcal{G}|$.

Proof. By the assumption, the size of $\Gamma_{\max }$ is $O(|\mathcal{G}|)$. Thus, the two loops terminate in $O(|\mathcal{G}|)$ iterations. At each iteration, $\operatorname{Expand}_{\mathcal{G}, \mathcal{B}}(\mathcal{G})$ and $\operatorname{Shrink}_{\mathcal{G}, \mathcal{B}}(\mathcal{G})$ can be computed in time $O(|\mathcal{G}|)[5$ hence the result.

Actually, we can use Rehof and Mogensen's algorithm [22] to accelerate the above algorithm, and obtain a linear time algorithm. (The idea is just to recompute Expand $_{\mathcal{G}, \mathcal{B}}$ and Shrink $_{\mathcal{G}, \mathcal{B}}$ only for variables whose relevant bindings were updated. As $\Gamma(x)$ is updated only a constant number of times for each variable $x$, and the number of typing bindings that are affected by the update is a constant, the resulting algorithm runs in time linear in $|\mathcal{G}|$.) More precisely, if the number of states of $\mathcal{B}$ is $|Q|$ and the largest arity of functions is $A$, the algorithm runs in time $O\left(|\mathcal{G}| \exp _{n}(p(A|Q|))\right.$, where $p(x)$ is a polynomial of $x$, and $\exp _{n}(x)$ is defined by: $\exp _{0}(x)=x, \exp _{k+1}(x)=2^{\exp _{k}(x)}$.

Soundness. The soundness of the algorithm follows immediately from that of Kobayashi's type system [12 (or Theorem 1): note that $\Gamma$ at the last line of the algorithm satisfies $\Gamma \subseteq \mathbf{S h r i n k}_{\mathcal{G}, \mathcal{B}}(\Gamma)$.

Theorem 4. If the algorithm outputs "yes", then $\llbracket \mathcal{G} \rrbracket$ is accepted by $\mathcal{B}^{\perp}$.

Completeness. A recursion scheme is in normal form if each rewrite rule is either of the form (i) $F \mapsto \lambda \widetilde{x}$.t where $t$ does not contain terminals, or (ii) $F \mapsto \lambda \widetilde{x} . a \widetilde{x}$. We show that the algorithm is complete when the given recursion scheme is in normal form 6 We now prove the completeness, i.e., if $\llbracket \mathcal{G} \rrbracket$ is accepted by $\mathcal{B}^{\perp}$, then the algorithm outputs "yes". From the game semantic intuition described in Remark 1, the reason for the completeness is intuitively clear: If a function behaves as described by a type $\theta$ in a reduction sequence of the given recursion scheme, then there should be a sequence of interactions between the function and the environment that conforms to $\theta$. As the sequence of interactions evolves, the

${ }^{5}$ To guarantee this, we need to normalize the rewrite rules of $\mathcal{G}$ in advance [16, so that the size of body $\mathcal{R}(F)$ of each non-terminal $F$ is bounded by a constant.

${ }^{6}$ Note that this does not lose generality, as we can always transform a recursion scheme into an equivalent recursion scheme in normal form before applying the algorithm, by introducing the rule $A \mapsto \lambda \widetilde{x} . a \widetilde{x}$ for each terminal $a$, and replace all the other occurrences of $a$ with $A$. We conjecture that the algorithm is complete without the normal form assumption. 
function's behavior should gradually evolve from $\top \rightarrow \cdots \rightarrow \top \rightarrow q$ (which represents a state where the environment has just called the function to ask for a tree of type $q$ ) to $\top \rightarrow \cdots \rightarrow\left(\top \rightarrow \cdots \rightarrow \top \rightarrow q^{\prime}\right) \rightarrow \cdots \rightarrow \top \rightarrow q$ (which represents a state where the function has responded to ask the environment to provide a tree of type $q^{\prime}$ ), and eventually to $\theta$. Such evolution of the function's type can be computed by $\operatorname{Expand}_{\mathcal{G}, \mathcal{B}}$, and $\theta$ should be eventually generated.

The actual proof is however rather involved. We defer the proof to the extended version [14 and just state the theorem here.

Theorem 5. Suppose $\mathcal{G}$ is in normal form. If $\llbracket \mathcal{G} \rrbracket$ is accepted by $\mathcal{B}^{\perp}$, then the algorithm outputs "yes".

\section{Experiments}

We have implemented the new model checking algorithm, and tested it for several recursion schemes. The result of the preliminary experiments is shown in Table1. The experiments were conducted on a machine with $\operatorname{Intel}(\mathrm{R}) \mathrm{Xeon}(\mathrm{R}) \mathrm{CPU}$ with $3 \mathrm{Ghz}$ and $8 \mathrm{~GB}$ memory. More information about the benchmark is available at http://www.kb.ecei.tohoku.ac.jp/ koba/gtrecs/

The column "order" shows the order of the recursion scheme. The columns "hybrid" and "new" show the running times of the hybrid and new algorithms respectively, measured in seconds. The cell marked by "-" in the column "hybrid" shows that the hybrid algorithm has timed out (where the time limit is $10 \mathrm{~min}$.) or run out of stack. The columns " $\Gamma_{1}$ " and " $\Gamma_{2}$ " show the numbers of atomic types in the type environment $\Gamma$ after the first and second loops of the new algorithm.

The table on the lefthand side shows the result for the following recursion scheme $\mathcal{G}_{n, m}[13$ :

$$
\begin{aligned}
& \left\{S \mapsto F_{0} G_{n-1} \cdots G_{2} G_{1} G_{0},\right. \\
& F_{0} \mapsto \lambda f \cdot \lambda \widetilde{x} \cdot F_{1}\left(F_{1} f\right) \widetilde{x}, \cdots, F_{m-1} \mapsto \lambda f \cdot \lambda \widetilde{x} \cdot F_{m}\left(F_{m} f\right) \widetilde{x}, F_{m} \mapsto \lambda f \cdot \lambda \widetilde{x} \cdot G_{n} f \widetilde{x}, \\
& \left.G_{n} \mapsto \lambda f \cdot \lambda z \cdot \lambda \cdot \widetilde{x} \cdot f(f z) \widetilde{x}, \cdots, G_{2} \mapsto \lambda f \cdot \lambda z \cdot f(f z), G_{1} \mapsto \lambda z \cdot \mathrm{a} z, G_{0} \mapsto c\right\}
\end{aligned}
$$

$S$ is reduced to $\mathrm{a}^{\exp _{n}(m)}\left(G_{0}\right)$ and then to $\mathrm{a}^{\exp _{n}(m)}(\mathrm{c})$. The verified property is that the number of a is even. The hybrid algorithm [10] requires $O\left(\exp _{n}(m)\right)$ expansions to extract the type information on $G_{0}$, so that it times out except for the case $n=3, m=1$. In contrast, the new algorithm works even for the case $n=4, m=10$. For a fixed $n$, the size of type environments (the columns $\Gamma_{1}$ and $\Gamma_{2}$ ) is almost linear in $m$. The running times are not linear in $m$ due to the naïveness of the current implementation, but exponential slowdown with respect to $m$ is not observed. As expected, the sizes of type environments (the columns $\Gamma_{1}$ and $\Gamma_{2}$ ) are much smaller than that of $\Gamma_{\max }$. For $\mathcal{G}_{3,1}$, the size of $\Gamma_{\max }$ is about $3 \times 2^{2057}$, so that the naïve algorithm does not work even for $\mathcal{G}_{3,1}$.

In the table on the righthand side, Example1 is the recursion scheme given in Example 1, where the trivial automaton is given in Example 2. The recursion schemes Twofiles - Lock2 have been taken from the benchmark set used in 
Table 1. The result of experiments. Times are in seconds.

\begin{tabular}{|l|r|r|r|r|r|}
\hline & order & hybrid & new & $\Gamma_{1}$ & $\Gamma_{2}$ \\
\hline $\mathcal{G}_{3,1}$ & 3 & 0.002 & 0.021 & 61 & 41 \\
\hline $\mathcal{G}_{3,5}$ & 3 & - & 0.135 & 161 & 97 \\
\hline $\mathcal{G}_{3,10}$ & 3 & - & 0.382 & 286 & 167 \\
\hline $\mathcal{G}_{4,1}$ & 4 & - & 0.563 & 302 & 206 \\
\hline $\mathcal{G}_{4,5}$ & 4 & - & 14.856 & 1079 & 703 \\
\hline $\mathcal{G}_{4,10}$ & 4 & - & 43.815 & 2054 & 1328 \\
\hline
\end{tabular}

\begin{tabular}{|l|r|r|r|r|r|}
\hline & order & hybrid & new & $\Gamma_{1}$ & $\Gamma_{2}$ \\
\hline Example1 & 2 & 0.002 & 0.002 & 15 & 13 \\
\hline Twofiles & 3 & 0.001 & 0.228 & 468 & 187 \\
\hline FileWrong & 3 & 0.001 & 0.116 & 398 & 142 \\
\hline File0camlc & 3 & 0.003 & 1.162 & 1610 & 414 \\
\hline Lock2 & 3 & 0.013 & 98.785 & 2464 & 1191 \\
\hline Nondet & 3 & N.A. & 0.013 & 77 & 63 \\
\hline
\end{tabular}

[10, obtained by encoding the resource usage verification problems 12 . We have used the refined encoding given in [15] however 7 Unfortunately, for these recursion schemes, the new algorithm is slower than the hybrid algorithm by several orders of magnitude. Further optimization is required to see whether this is a fundamental limitation of the new algorithm. Finally, Nondet is an order-3 recursion scheme that generates a tree representation of an infinite list $[(0,1) ;(1,2) ;(2,3) ; \cdots]$, where each natural number $n$ is represented by the tree $s^{n}(z)$. A non-deterministic trivial automaton is used for expressing the property that each pair in the list is either a pair of an even number and an odd number, or a pair of an odd number and an even number. Our new algorithm works well, while the hybrid algorithm (which works only for deterministic trivial automata) is not directly applicable 8

\section{Related Work}

We have already discussed the main related work in Section 11 There are several algorithms for the model checking of higher-order recursion schemes (some of which are presented in the context of showing the decidability). Besides those already mentioned [2012 10 16], Hague et al. 6] reduce the modal $\mu$-calculus model checking to a parity game over the configuration graph of a collapsible pushdown automaton. Aehlig [2] gives a trivial automata model checking algorithm based on a finite semantics, which runs in a fixed-parameter non-deterministic linear time in the size of the recursion scheme. For recursion schemes with the so called safety restriction, Knapik et al. 9] give another decision procedure, which reduces a model checking problem for an order- $n$ recursion scheme to that for an order$(n-1)$ recursion scheme. As mentioned already, however, the only practical previous algorithm (which was ever implemented) is Kobayashi's hybrid algorithm [10], to our knowledge. Its worst-case complexity is hyper-exponential in the size of recursion schemes, unlike our new algorithm. Recently, Lester et al. [19] extended

\footnotetext{
7 The encoding in 12 produces order- 4 recursion schemes, while that of 15 produces order-3 recursion schemes. An additional optimization is required to handle the encoding of [12]: see [14.

${ }^{8}$ As mentioned in Section 6, Lester et al. 19] recently extended the hybrid algorithm to deal with alternating Büchi automata.
} 
the hybrid algorithm to deal with alternating Büchi automata. As the basic mechanism for collecting type information remains the same, their algorithm also suffers from the same worst-case behavior as Kobayashi's hybrid algorithm.

As mentioned already, though our new algorithm is type-based, it has been inspired from game semantics [120]. In the previous type-based approach [12], the types of a function provide coarse-grained information about the function's behavior, in the sense that the types tell us information about complete runs of the function. On the other hand, the game-semantic view provides more finegrained information, about partial runs of a function. For example, $F: \top \rightarrow q_{0}$ belonging to the least fixedpoint of $\operatorname{Expand}_{\mathcal{G}, \mathcal{B}}$ means that $F$ may be called in a context where a tree of type $q_{0}$ is required, not necessarily that $F$ returns a tree of type $q_{0}$ for arbitrary arguments. This enabled us to collect type information by a least fixedpoint computation, yielding a realistic linear time algorithm.

As explained in Section 2, the model checking of higher-order recursion schemes has been reduced to the type checking problem for an intersection type system. Thus, our algorithm may have some connection to intersection type inference algorithms [7/5]. The connection is however not so clear. To our knowledge, the existing inference algorithms have a process corresponding to $\beta$-normalization [5], so that even for terms without recursion, the worst-case complexity of intersection type inference is non-elementary in the program size.

\section{Conclusion}

Studies of the model checking of higher-order recursion schemes have started from theoretical interests [89], but it is now becoming the basis of automated verification tools for higher-order functional programs [12 18,17/21]. Thus, it is very important to develop an efficient model checker for higher-order recursion schemes. The new algorithm presented in this paper is the first one that is efficient both in theory (in the sense that it is fixed-parameter linear time) and in practice (in the sense that it is runnable for recursion schemes of order 3 or higher). The practical efficiency is however far from satisfactory (recall Section (5), so that further optimization of the algorithm is necessary. As the structure of the new algorithm is simple, we expect that it is more amenable to various optimization techniques, such as BDD representation of types. A combination of the hybrid and new algorithms also seems useful. It does not seem so difficult to extend the new algorithm to obtain a practical fixed-parameter polynomial time algorithm for the full modal $\mu$-calculus; It is left for future work.

\section{References}

1. Abramsky, S., McCusker, G.: Game semantics. In: Computational Logic: Proceedings of the 1997 Marktoberdorf Summer School, pp. 1-56. Springer, Heidelberg (1999)

2. Aehlig, K.: A finite semantics of simply-typed lambda terms for infinite runs of automata. Logical Methods in Computer Science 3(3) (2007) 
3. Ball, T., Rajamani, S.K.: The SLAM project: Debugging system software via static analysis. In: Proc. of POPL, pp. 1-3 (2002)

4. Beyer, D., Henzinger, T.A., Jhala, R., Majumdar, R.: The software model checker Blast. International Journal on Software Tools for Technology Transfer 9(5-6), 505-525 (2007)

5. Carlier, S., Polakow, J., Wells, J.B., Kfoury, A.J.: System E: Expansion variables for flexible typing with linear and non-linear types and intersection types. In: Schmidt, D. (ed.) ESOP 2004. LNCS, vol. 2986, pp. 294-309. Springer, Heidelberg (2004)

6. Hague, M., Murawski, A., Ong, C.-H.L., Serre, O.: Collapsible pushdown automata and recursion schemes. In: Proceedings of 23rd Annual IEEE Symposium on Logic in Computer Science, pp. 452-461. IEEE Computer Society, Los Alamitos (2008)

7. Kfoury, A.J., Wells, J.B.: Principality and type inference for intersection types using expansion variables. Theor. Comput. Sci. 311(1-3), 1-70 (2004)

8. Knapik, T., Niwinski, D., Urzyczyn, P.: Deciding monadic theories of hyperalgebraic trees. In: Abramsky, S. (ed.) TLCA 2001. LNCS, vol. 2044, pp. 253-267. Springer, Heidelberg (2001)

9. Knapik, T., Niwinski, D., Urzyczyn, P.: Higher-order pushdown trees are easy. In: Nielsen, M., Engberg, U. (eds.) FOSSACS 2002. LNCS, vol. 2303, pp. 205-222. Springer, Heidelberg (2002)

10. Kobayashi, N.: Model-checking higher-order functions. In: Proceedings of PPDP 2009, pp. 25-36. ACM Press, New York (2009), see also [13]

11. Kobayashi, N.: TRECS (2009), http://www.kb.ecei.tohoku.ac.jp/ koba/trecs/

12. Kobayashi, N.: Types and higher-order recursion schemes for verification of higherorder programs. In: Proc. of POPL, pp. 416-428 (2009), see also [13]

13. Kobayashi, N.: Model checking higher-order programs. A revised and extended version of [12] and [10], available from the author's web page (2010)

14. Kobayashi, N.: A practical linear time algorithm for trivial automata model checking of higher-order recursion schemes (2010), an extended version http://www.kb.ecei.tohoku.ac.jp/ koba/gtrecs/

15. Kobayashi, N., Ong, C.-H.L.: Complexity of model checking recursion schemes for fragments of the modal mu-calculus. In: Albers, S., Marchetti-Spaccamela, A., Matias, Y., Nikoletseas, S., Thomas, W. (eds.) ICALP 2009. LNCS, vol. 5556, pp. 223-234. Springer, Heidelberg (2009)

16. Kobayashi, N., Ong, C.-H.L.: A type system equivalent to the modal mu-calculus model checking of higher-order recursion schemes. In: Proceedings of LICS 2009, pp. 179-188. IEEE Computer Society Press, Los Alamitos (2009)

17. Kobayashi, N., Sato, R., Unno, H.: Predicate abstraction and cegar for higher-order model checking (July 2010) (unpublished manuscript)

18. Kobayashi, N., Tabuchi, N., Unno, H.: Higher-order multi-parameter tree transducers and recursion schemes for program verification. In: Proc. of POPL, pp. 495-508 (2010)

19. Lester, M.M., Neatherway, R.P., Ong, C.-H.L., Ramsay, S.J.: Model checking liveness properties of higher-order functional programs (2010) (unpublished manuscript)

20. Ong, C.-H.L.: On model-checking trees generated by higher-order recursion schemes. In: LICS 2006, pp. 81-90. IEEE Computer Society Press, Los Alamitos (2006)

21. Ong, C.-H.L., Ramsay, S.: Verifying higher-order programs with pattern-matching algebraic data types. In: Proceedings of POPL 2011 (to appear, 2011)

22. Rehof, J., Mogensen, T.: Tractable constraints in finite semilattices. Science of Computer Programming 35(2), 191-221 (1999) 